\title{
Experimental Study and Computational Simulation for Shock Characteristics Estimation of Okinawa's Soils "Jahgaru"
}

\author{
Y Higa1*, H lyama², K Shimojima', \\ M Nishi², $\mathbf{S}$ Itoh $^{3}$
}

1. Department of Mechanical Systems Engineering, National Institute of Technology, Okinawa College, Japan

2. Department of Mechanical and Intelligent Systems Engineering, National Institute of Technology, Kumamoto College, Japan.

3. Institution of Pulsed Power Science, Kumamoto University, Kumamoto, Japan

\begin{abstract}
To clarify the shock characteristics of Okinawa's unique soils "Jahgaru" that is widely distributed in southern part of Okinawa Main Island, an experimental investigation of dynamics properties such as shockwave propagation, pressure and particle velocity have been performed using impedance matching method. Therefore, we have also obtained the Hugoniot date of "Jahgaru". And then, to reveal a validity of the material characteristics, a computational model for the experimental procedure using ALE simulation have been developed. A comparison between numerical results and experimental ones, the capability of proposed method through the numerical simulations have been confirmed.
\end{abstract}

\section{INTRODUCTION}

The main objective of our research is to contribute to a technique of unexploded bomb (UXB) disposal such as an establishment of evacuation areas based on the computational mechanics. The explosion characteristics of UXB depends on the bombing case, explosives and surrounding environments such as air, soil, construction and building. To our knowledge, however, the dynamic characteristics of Okinawa's soil has not reported yet though the static characteristics has few reported. Thereby, it is significantly important to clarify the dynamic characteristics of the soil. The types of soil in Okinawa Island are classified into four major groups [1] as follows: (a) Kunigami Mahji, (b) Shimajiri Mahji (dark-red soil), (c) Jahgaru (grey terrace soil) and (d) Kucha (alluvial soil). Kunigami Mahji is widely distributed in Northern place in Okinawa Island. Shimajiri Mahji and Jahgaru is also distributed in Southern place. Kucha meaning of clay in the local Okinawan language is distributed along the coastline. In our research, we focused on the dynamic soil characteristics of the Jahgaru because the soil widely distributed in the Southern part of Okinawa where the UXB is discovered well. It is very important to reveal the dynamic characteristics of Jahgaru to predict the fragment behavior of the soil at the blast. Thus, in

*Corresponding Author: y.higa@okinawa-ct.ac.jp 
order to clarify the shock characteristics of the Jahgaru, an experimental investigation of dynamics properties have been performed using impedance matching method [2,3]. In addition to verify the validity of the material properties, a computational model corresponding to the experimental procedure using ALE simulation have been developed.

\section{EXPERIMENTAL PROCEDURE}

To clarify the dynamics characteristic of the Okinawa's unique soil i.e. Jahgaru, the optical observation using the shadowgraph system [4-9] have been performed to evaluate the shock wave induced by the detonation. The large number of studies using shadowgraph method for shock wave visualization has been reported in the past. The shadowgraph system consists of an image converter camera (IMACON468, 20Mfps, 1ns/mm) and Xenon lamp (HL20/50 type flash-right, output 500J) were used to observe the incident / transmit shock wave propagation behavior in acrylic resin (Polymethyl methacrylate; PMMA). The observation system in this study has the dimension and consists of an electric detonator (ED), detonation fuse (DF), explosive lens (SEP+HABW), main explosive (SEP), explosive container (PVC), gap and base block (PMMA), and Jahgaru as shown in Fig. 1.

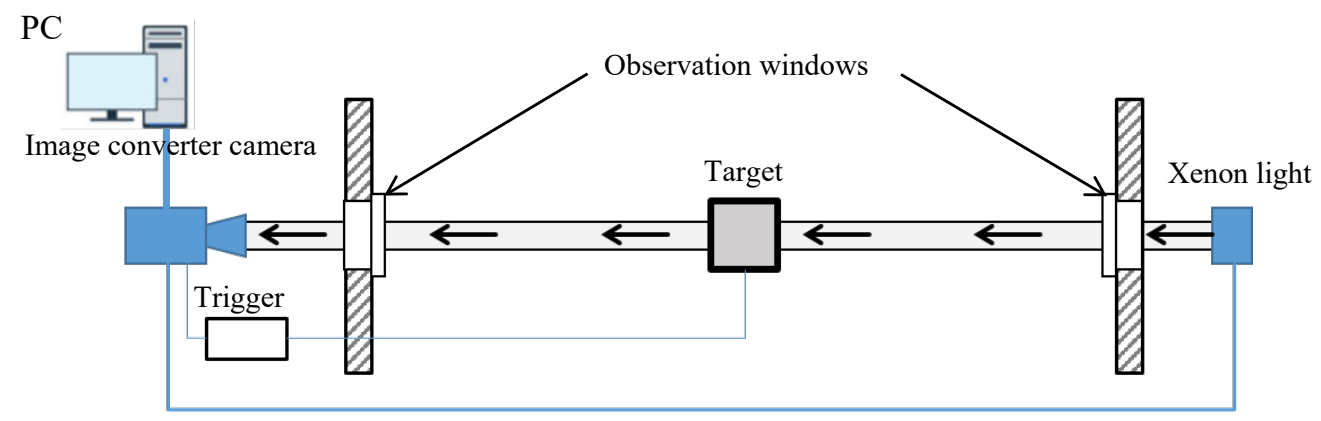

Figure 1. Schematic illustration of optical measurement system

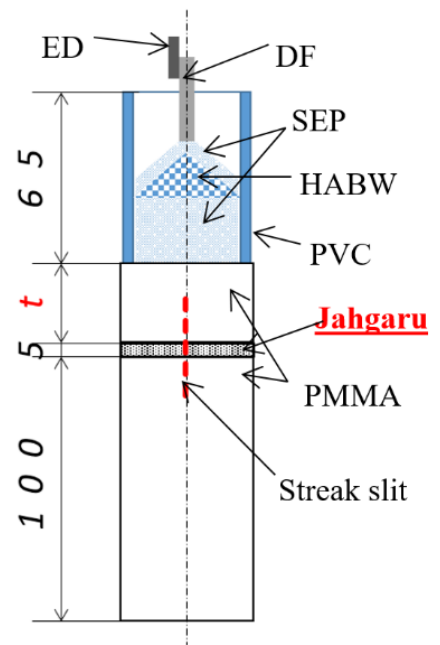

Figure 2. Schematic illustration and dimension of an optical observation 
The explosive lens is set on the top of the main explosive (SEP; detonation velocity $6980 \mathrm{~m} / \mathrm{s}$, density $1310 \mathrm{~kg} / \mathrm{m}^{3}$ ) and corn shape explosive (HABW; detonation velocity $4750 \mathrm{~m} / \mathrm{s}$, density $2200 \mathrm{~kg} / \mathrm{m}^{3}$ ) to make a planner wave. We can prepare the different transmitted velocity depending on the length of gap block. The Hugoniot equation of state between shock velocity Us and particle velocity up has calculated with impedance matching method [2,3]. These relationship between already known (PMMA) and unknown transmitted velocity (Jahgaru) has obtained the shock Hugoniot data of Jahgaru. In this experiment, the thickness ' $t$ ' of PMMA gap as shown in Fig. 2 were 10, 30 and 50mm, respectively.

\section{COMPUTATIONAL MODELING OF THE EXPERIMENTAL SETUP}

To verify the validity of the dynamics characteristic of Jahgaru, the computational model which is arranged as well as the experimental setup has been constructed using a commercial finite element software (ANSYS Autodyn) and has been performed introducing the dynamic characteristics obtained by the experimental results as shown in Fig. 3. In this study, the explosive (SEP and HABW), PVC, PMMA and Jahgaru are discretized by ALE finite elements [10].

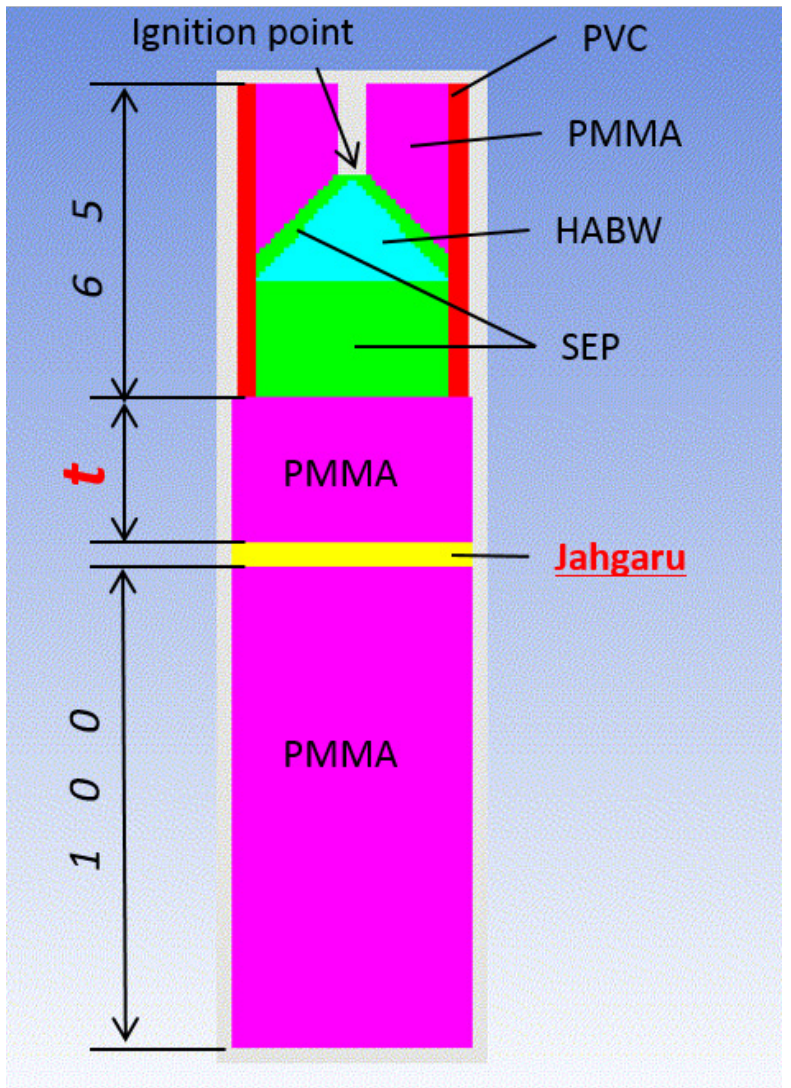

Figure 3. Computational model 


\subsection{Equation of state.}

The equation of state for an explosive in this study was used Jones-Wilkins-Lee (JWL) types equation [11].

$$
\begin{gathered}
P=\mathrm{A}\left[1-\frac{\omega}{R_{1} V}\right] e^{-R_{1} V}+B\left[1-\frac{\omega}{R_{2} V}\right] e^{-R_{2} V}+\frac{\omega E}{V} . \\
P_{C J}=\frac{\rho_{0} D^{2}}{\gamma+1}, \quad \gamma=c_{p} / c_{v}
\end{gathered}
$$

where $A, B, R_{1}, R_{2}$, and $\omega$ are the JWL parameters; V is the ratio of the volume of the gases produced by the explosion to the initial volume of the undetonated explosive; $\mathrm{E}$ is the detonation energy per unit volume and has an initial value of $\mathrm{E}_{0} ; P_{C J}$ is the Chapman-Jouguet pressure, which depends on the initial density $\rho_{0}$ of the explosive and the detonation velocity $\mathrm{D}$; and $\gamma$ is the isentropic expansion factor. JWL parameters of SEP and HABW are given in Table.1.

Table 1. JWL parameters for HABW and SEP explosive

\begin{tabular}{lllllllll}
\hline \multirow{2}{*}{ Explosive } & $\begin{array}{l}\boldsymbol{P}_{\boldsymbol{C J}} \\
{[\mathbf{G P a}]}\end{array}$ & $\begin{array}{l}\mathbf{D} \\
{[\mathbf{m} / \mathbf{s}]}\end{array}$ & $\begin{array}{l}\boldsymbol{\rho}_{\mathbf{0}} \\
{\left[\mathbf{k g} / \mathbf{m}^{3}\right]}\end{array}$ & $\begin{array}{l}\mathbf{A} \\
{[\mathbf{G P a}]}\end{array}$ & $\begin{array}{l}\mathbf{B} \\
{[\mathbf{G P a}]}\end{array}$ & $\mathbf{R}_{\mathbf{1}}$ & $\mathbf{R}_{\mathbf{2}}$ & $\boldsymbol{\omega}$ \\
\hline HABW & 12.4 & 4750 & 2200 & 835 & 12.67 & 5.85 & 1.53 & 0.247 \\
SEP & 15.9 & 6980 & 1310 & 365 & 2.31 & 4.3 & 1.0 & 0.28 \\
\hline
\end{tabular}

The hydrostatic pressure $p$ was calculated for PVC, PMMA and Jahgaru using the MieGrüneisen equation of state (EOS) [12], which is given as

$$
p=\frac{\rho_{0} c_{0}^{2} \eta}{(1-s \eta)^{2}}\left[1-\frac{\Gamma_{0} \eta}{2}\right]+\Gamma_{0} \rho_{0} e .
$$

where $\rho_{0}$ is the initial density, $\mathrm{c}_{0}$ is the acoustic velocity, $\Gamma_{0}$ is a Grüneisen constant and $s$ is a linear Hugoniot slope coefficient, $e$ is the specific internal energy and $\eta=1-\rho_{0} / \rho$ is a material parameter defined by the material density $\rho$. The values of these parameters for the pertinent materials are given in Table 2. Here the material characteristic of Jahgaru is calculated from an experimental result addressed after this paragraph.

Table 2. Material constants in Mie-Grüneisen EoS

\begin{tabular}{lllll}
\hline & $\boldsymbol{\rho}_{0}$ & $\mathbf{c}_{\mathbf{0}}$ & $\mathbf{s}[-]$ & $\boldsymbol{\Gamma}[-]$ \\
\hline PVC & {$\left[\mathbf{k g} / \mathbf{m}^{3}\right]$} & {$[\mathbf{m} / \mathbf{s}]$} & & \\
PMMA & 1380 & 2300 & 1.47 & 0.4 \\
\hline
\end{tabular}




\section{RESULTS AND DISCUSSIONS}

4.1. Calculation of Jahgaru's Hugoniot EOS.

Figure 4 shows the streak photograph and the schematic diagram to calculate a) the incident and b) transmitted shock wave velocity, in case of PMMA gap $\mathrm{t}=50 \mathrm{~mm}$. the both curves are approximated by nonlinear curve function as follows:

$$
\begin{gathered}
L=A_{1}\left\{1-\exp \left(-B_{1} t\right)\right\}+A_{2}\left\{1-\exp \left(-B_{2} t\right)\right\} \\
+A_{3}\left\{1-\exp \left(-B_{3} t\right)\right\}+c_{0} t .
\end{gathered}
$$

where $A_{1}, A_{2}, A_{3}, B_{1}, B_{2}$ and $B 3$ material parameters. $c_{0}$ denotes the acoustic velocity of PMMA. These summaries of an analytical result have been summarized as shown in Table 3. Therefore, the relationship between the Jahgaru's particle velocity and Rankin-Hugoniot EOS derive the shock characteristics of Jahgaru as shown in Table 3. The figure 5 shows Hugoniot EOS of Jahgaru corresponding to the relationship between the particle velocities $U_{s}$ and $u_{p}$. In this study, we can summarize the dynamic characteristics of Jahgaru as follows: the acoustic velocity $\mathrm{c}_{0}=0.701 \mathrm{~km} / \mathrm{s}$, and Hugoniot's slope coefficient $\mathrm{s}=2.919$.

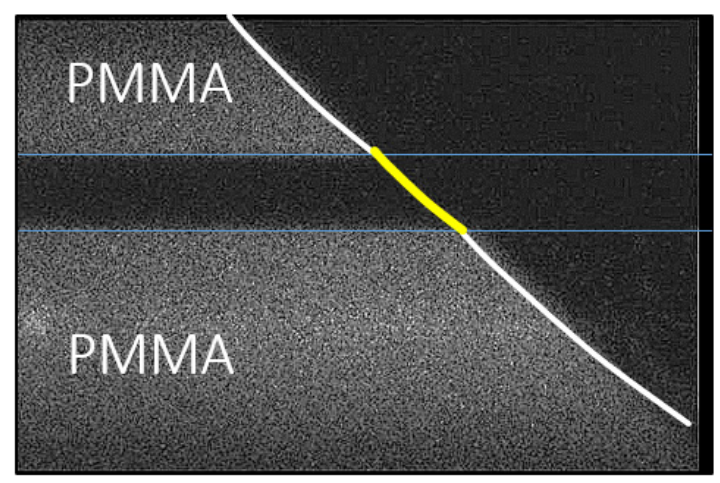

\section{Streak photograph of S.W.}

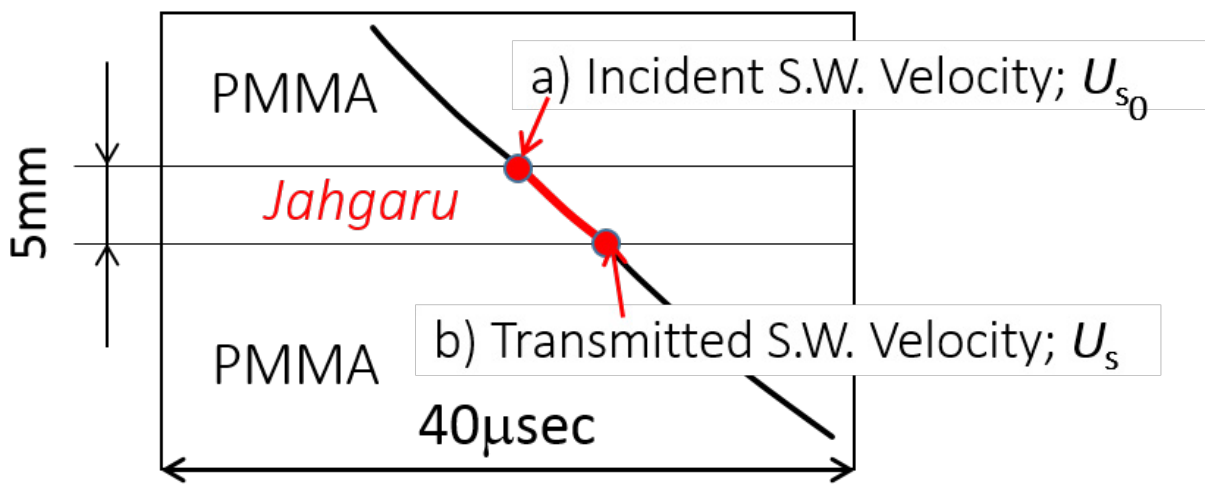

Figure 4.Streak photograph of shock wave and schematic illustration of a) incident shock wave velocity and b) transmitted shock wave velocity 
Table 3. Experimental results for Jahgaru

\begin{tabular}{|c|c|c|c|c|}
\hline $\begin{array}{l}\text { PMMA } \\
\text { Gap } \\
\text { t, mm }\end{array}$ & $\begin{array}{l}\text { PMMA-Jahgaru } \\
\text { a)Incident velocity } \\
\mathrm{U}_{\mathrm{s} 0}, \mathrm{~km} / \mathrm{s}\end{array}$ & $\begin{array}{l}\text { Jahgaru-PMMA } \\
\text { b)Transmitted } \\
\text { velocity } U_{\mathrm{s}}, \mathrm{km} / \mathrm{s}\end{array}$ & $\begin{array}{l}\text { Particle } \\
\text { velocity } \\
\mathbf{u}_{\mathrm{p}}, \mathbf{k m} / \mathbf{s}\end{array}$ & $\begin{array}{l}\text { Pressure } \\
\text { p ,GPa }\end{array}$ \\
\hline 10 & 3.74 & 3.52 & 0.966 & 6.85 \\
\hline 10 & 3.61 & 3.32 & 0.898 & 6.32 \\
\hline 30 & 2.65 & 2.34 & 0.651 & 1.81 \\
\hline 30 & 2.74 & 2.33 & 0.643 & 1.82 \\
\hline 50 & 2.37 & 1.94 & 0.423 & 1.40 \\
\hline 50 & 2.43 & 2.07 & 0.471 & 1.86 \\
\hline
\end{tabular}

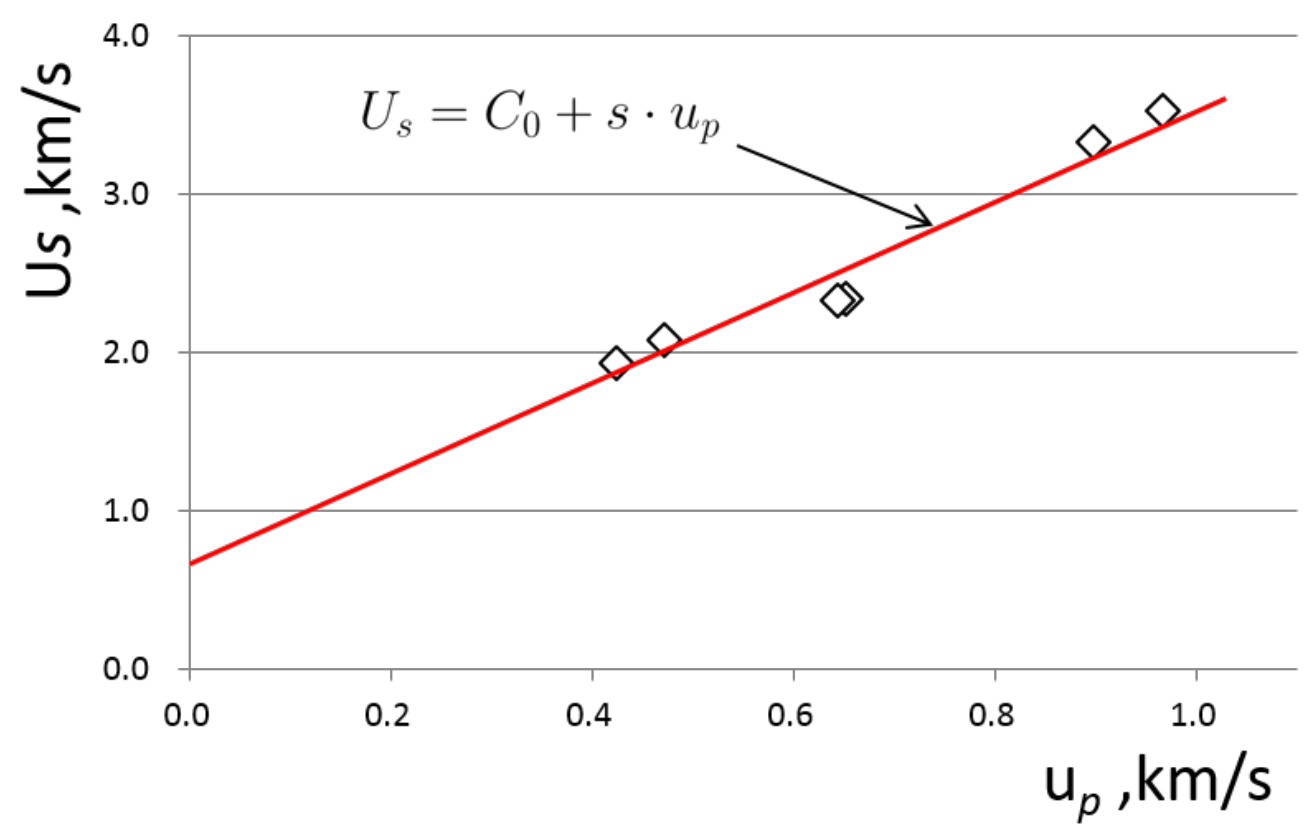

Figure 5. Hugoniot EOS of Jahgaru

\subsection{Computational simulation.}

To verify the validity of the dynamic characteristics, a computational model for the experimental procedure using ALE simulation have been developed as shown in Fig. 3 and the computational simulation introducing the Jahgaru's material parameters have been performed.

The pressure propagation behavior from the explosive initiation to transmitting shockwave in PMMA were shown in Fig. 6. It is seen that the front of the shock wave propagates and it is almost flat just before entering the PMMA ( $\mathrm{t}=8.05 \mathrm{~ns})$. This result suggests that the experimental setup could be obtained the quasi-plane wave necessary to discuss Hugoniot EOS using the explosive lens. The figure 7 shows the pressure history of gauge point \#1-\#6 as shown in this figure. The gauge point $\# 2$ corresponds to the transmitted velocity, Us, of Jahgaru-PMMA interface. In the case of PMMA gap length $\mathrm{t}=10$ and $30 \mathrm{~mm}$, the peak pressure at gauge \#2 are 7.16 and $2.02 \mathrm{GPa}$, respectively. The computational results are fairly good 
agreement with the experimental ones because the experimental results are 6.85 and $1.81 \mathrm{GPa}$. These results suggest that the dynamic characteristics of Jahgaru estimated from experimental outcomes is quite valid.

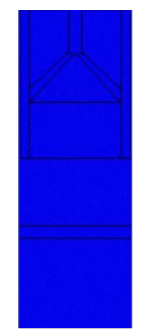

$0 \mathrm{~ns}$

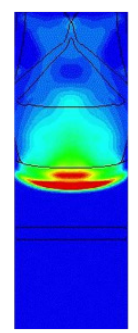

$10.7 \mathrm{~ns}$

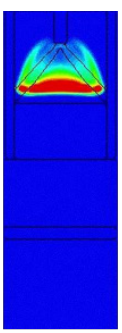

$3.66 \mathrm{~ns}$

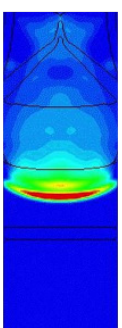

$12.1 \mathrm{~ns}$

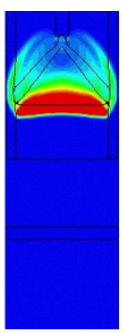

$5.27 \mathrm{~ns}$

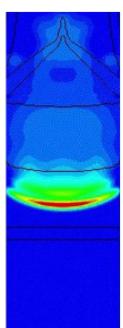

$12.9 \mathrm{~ns}$

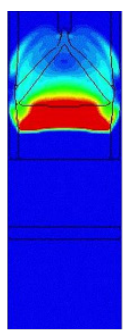

$6.20 \mathrm{~ns}$

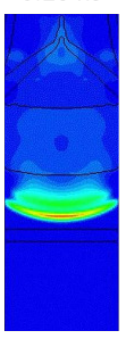

$14.3 \mathrm{~ns}$

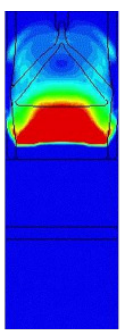

$7.05 \mathrm{~ns}$

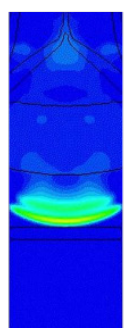

$15.0 \mathrm{~ns}$

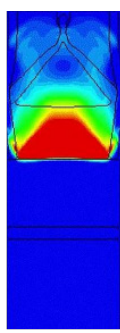

$8.05 \mathrm{~ns}$

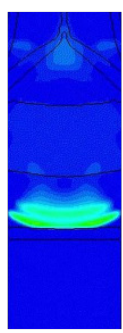

$16.1 \mathrm{~ns}$

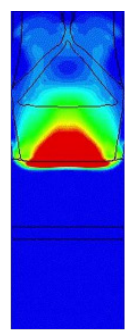

$8.86 \mathrm{~ns}$

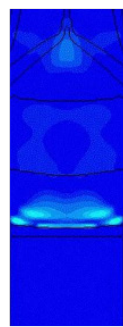

$17.8 \mathrm{~ns}$
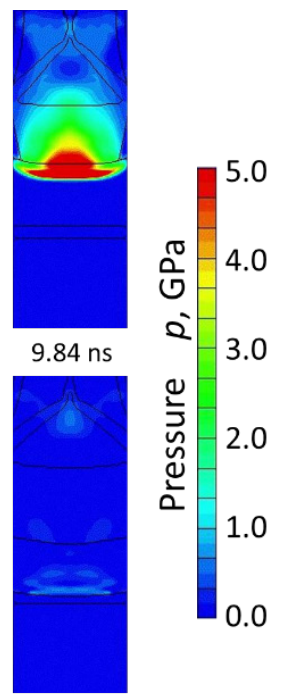

$18.7 \mathrm{~ns}$

Figure 6. Snap shots of pressure propagation behavior at each time steps. This result corresponds to PMMA gap length $\mathrm{t}=30 \mathrm{~mm}$.
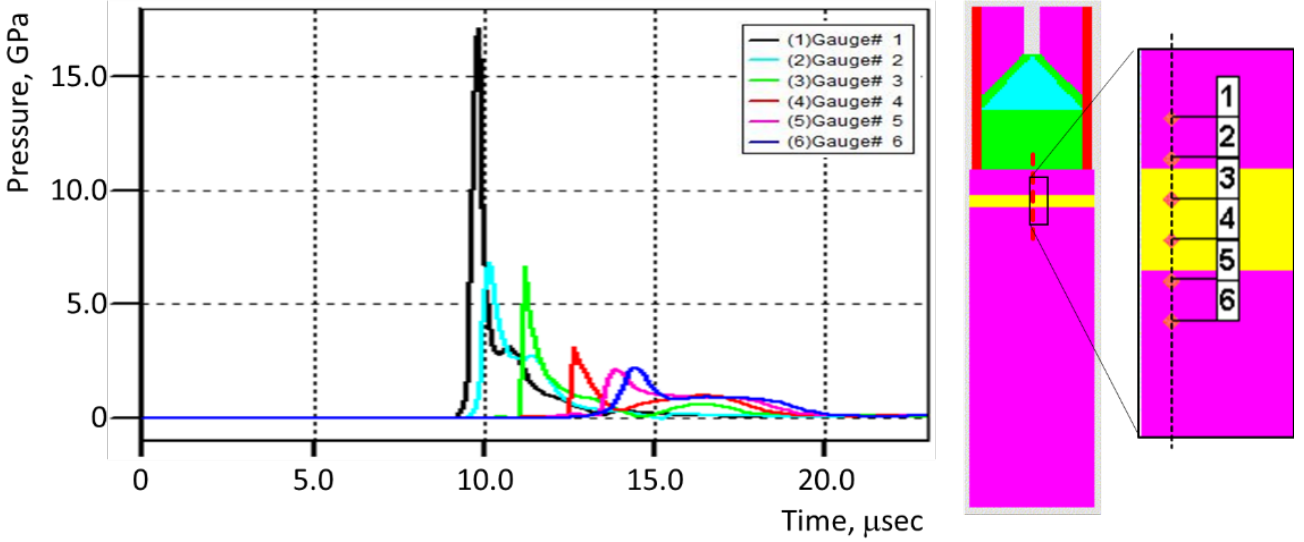

Figure 7. Pressure vs. time history at the pressure gauge \#1 - \#6. These results correspond to the PMMA gap length $t=10 \mathrm{~mm}$. 


\section{CONCLUSIONS}

To clarify the shock characteristics of Okinawa's unique soils "Jahgaru", an experimental investigation of dynamics properties such as shockwave propagation, pressure and particle velocity have been performed using impedance matching method. Therefore, we have also obtained the shock characteristics i.e. the Hugoniot date of "Jahgaru". And then, to reveal a validity of the material characteristics, a computational model for the experimental procedure using ALE simulation have been developed. A comparison between numerical results and experimental ones, the computational results are fairly good agreement with the experimental ones. These results suggest that the dynamic characteristics of Jahgaru estimated from experimental outcomes is quite valid.

\section{ACKNOWLEDGEMENT}

The authors gratefully acknowledge the support financial support from JSPS KAKENHI Grant-in-Aid for Scientific Research (C), JP16K01305. The experiment was conducted with the help of Prof. Shigeru TANAKA and the Explosion Experiment Facilities in Institution of Pulsed Power Science, Kumamoto University. We also would like to thank the joint research and the relationship.

\section{REFERENCES}

[1] S.S. Bakari, S.A. Vuai and A. Tokuyama: Forest soil fertility in Okinawa Island, subtropical region in Japan, Bulletin of the College of Science. Univ. Ryukyus: No.78, (2004), pp.267-284.

[2] H. Hornberg: Determination of Fume State Parameters from Expansion Measurements of Metal Tubes, Propellants, Explosives, Pyrotechnics Vol.11, Issue 1 (1986), pp.2331.

[3] I.F. Lan, S.C. Hung, W.M. Lin, C.Y. Chen, Y.M. Niu and J.H. Shiuan: A simple method of deducing JWL parameters from cylinder expansion test, Sci. Tech. Ener. Mat. Vol.53, No.3 (1992), pp149-157.

[4] S. Itoh, Z. Liu and Y. Nadamitsu: An investigation on the properties of underwater shock wave generated in underwater explosive of high explosive, ASME J. Pressure Vessel Technol. Vol.119 (1997), pp.498-502.

[5] A. Osada, H. Hamashima, Y. Kato and S. Itoh: Study on low velocity detonation phenomena in nitromethane, Int. J. Multiphysics Vol.3, No.1 (2009), pp.1-10.

[6] M. Otsuka, H. Maehara, M. Souli and S. Itoh: Study on development of vessel for shock pressure treatment for food, Int. J. Multiphysics Vol.1, No.1 (2007), pp.69-84.

[7] S. Irie, K. Greg, Z. Ren and S. Itoh: Dynamic property of aluminum foam, Int. J. Multiphysics, Vol.4, No.2 (2010), pp.103-111.

[8] O. Higa, R. Matsubara, K. Higa, et al.: Mechanism of the shock wave generation and energy efficiency by underwater discharge, Int. J. Multiphysics Vol.6, No.2 (2012), pp.89-97.

[9] O. Higa, A. Yasuda, Y. Higa, K. Shimojima, K. Hokamoto and S. Itoh: Optical examination of shockwave propagation induced by an underwater wire explosion, Int. J Multiphysics Vol.10, No.4 (2016), pp.343-353. 
[10] C.W. Hirt, A.A. Amsden and J.L. Cook: An Arbitrary Lagrangian Eulerian Computing Method for All Flow Speeds, J. Computational Physics Vol.14, No.3 (1974), pp.227253.

[11] C.L. Mader: Numerical Modeling of Detonations, University of California Press (1979).

[12] S.P. Marsh: LASL Shock Hugoniot Data, University of California Press, Berkeley (1980). 
